\title{
Gestural Attributions as Semantics in User Interface Sound Design
}

\author{
Kai Tuuri \\ Department of Computer Science and Information Systems \\ FI-40014 University of Jyväskylä, Finland \\ krtuuri@jyu.fi
}

Key words: gestures, user interfaces, sound design

\section{Cues of agency in communicative sounds}

Sound-based communication within different kinds of media have a long tradition. Sound design of radio-plays as early as 1920s have defined the basis for communicative use of sound effects which is still relevant in the tradition of film sound design. One of the common demands for sound designers have been the creation of sound effects with various emotional or intentional qualities. The suitable door knock for narration, for example, can be described as urging, gentle or angry in the manuscript [1]. This kind of agency perspective for sound design is widely adopted in the tradition of film sound design exploiting the natural perceptual bias towards looking for cues of agency in actions. But when it comes to the sound design for human-computer interaction (HCI), this kind of expressiveness is seldom used.

In this paper we argue that the design of non-speech audio feedback for the HCI context would vastly benefit from such agency approach, which aims at utilising the gestures of interpersonal interaction in the sonic interaction design. In the social interaction, we naturally express our mental states with bodily involvement which can be called either facial, vocal, hand or other kind of gestural action. As a communicative acts, gestures usually posses derived intentionality which is caused by the mental states of an acting person [2]. The involvement of gestural imagery in music perception[3], for example, suggests that similar motor-mimetic gestural attuning to the sound would be central element also in both the perception and the design of UI sounds. Therefore we suggest that gestural attributions of derived intentionality with sounds would be considered as a form of semantics and thus as means to design communicative sounds for interaction.

\section{What kind of semantics are gestural semantics?}

The meanings evoked by motor-mimetic (gestural) attuning to the sound are action-orientated and non-linguistic in nature. They refer to sensory-motoric 
experiences that we can imagine doing or feeling ourselves and thus can empathetically denote the intentional and emotional states by mentally imitating the perceived motor activity. Such attribution of intentionality can also be extended to the perception of material things if we can imagine (by imitation) the action being caused by the movement of an intentional subject. [4] Gestural attributions can thus imply various types of meanings. Those attributions can relate to any kind of bodily involvement providing, e.g., cues of communicative purpose of an act or cues of intentions and emotions in general. However, it is essential to keep in mind that the understanding of these attributions are observer-dependent and bound with the situational context.

\section{The role of gestures in sound design for interaction}

When the sound designer puts oneself into the context of interaction - the "dialogue" with user and machine - the designer can conceive her role as a person who is talking to the user. She can imagine participating that process, although in reality that occurs via the mediation of machine in use. However, from that imaginary perspective, the designer can experience what would be the proper means to communicate with a user, and how she could do it in that use situation. Following this line of reasoning and using the terminology of John R. Searle, the design of certain communicative sound can be conceived as an illocutionary act, counting not just speech acts but any bodily action that is motivated by intention to be understood in interaction. We argue that gestural imagery involved in such acts can form a basis for sound design, if these gestural mental images can be somehow attributed in result of the design. One way to do this is the physical articulation of the gestural imagery. Such articulation can be musical expression, vocal act, or any physical gesture that can produce acoustic resonances. Physical articulation of gestural imagery thus provides derived attributions of the corresponding motivation to communicate, in the form of concrete physical features to be utilised in the design. This method of using gestures in sound design has already demonstrated its effectiveness in real-world sound design cases [5].

\section{References}

1. Mott, R. L. Sound effects: Radio, TV and Film. Boston, MA: Focal Press (1990)

2. Searle, J. R. Intentionality: An Essay in the Philosophy of Mind. New York, NY: Cambridge University Press (1983)

3. Godøy, R. I. Gestural Imagery in the Service of Musical Imagery. In: Camurri, A. and Volpe, G. (eds.): Gesture-Based Communication in Human-Computer Interaction: 5th In-ternational Gesture Workshop, GW 2003, Volume LNAI 2915, Springer-Verlag, Berlin/Heidelberg (2004) 55-62

4. Leman, M. Embodied Music Cognition and Mediation Technology. Cambridge, MA: MIT Press (2008)

5. Tuuri, K., Pirhonen, A. and Eerola, T. Design and Evaluation of Prosody Based Non-Speech Audio Feedback for Mobile Training Application. Submitted paper. (2008) 